Revue musicale OICRM

\title{
La fabrique de la musique. Des processus créateurs à la médiation des musiques
}

\section{Hyacinthe Ravet}

Volume 7, numéro 2, 2020

Fenêtre ouverte sur la médiation de la musique

URI : https://id.erudit.org/iderudit/1072413ar

DOI : https://doi.org/10.7202/1072413ar

Aller au sommaire du numéro

Éditeur(s)

Observatoire interdisciplinaire de création et recherche en musique (OICRM)

ISSN

2368-7061 (numérique)

Découvrir la revue

Citer cet article

Ravet, H. (2020). La fabrique de la musique. Des processus créateurs à la médiation des musiques. Revue musicale OICRM, 7(2), 23-35.

https://doi.org/10.7202/1072413ar
Résumé de l'article

Comment appréhender la fabrication de la musique lorsque l'on cherche à la médier ? En quoi l'identification des différent·e-s acteur-rice-s qui participent à l'élaboration d'une oeuvre donne-t-elle une prise sur ce qu'est une " oeuvre " et ce qu'elle représente pour qui la reçoit ? Comment peut se situer un·e médiateur-rice dans cette activité de création, passée ou présente ? Cet article pose la médiation comme une activité partie prenante des processus créateurs en jeu. 


\title{
La fabrique de la musique. Des processus créateurs à la médiation des musiques
}

\author{
Hyacinthe Ravet
}

\begin{abstract}
Résumé
Comment appréhender la fabrication de la musique lorsque l'on cherche à la médier ? En quoi l'identification des différent·es acteur-rice's qui participent à l'élaboration d'une œuvre donne-t-elle une prise sur ce qu'est une " œuvre " et ce qu'elle représente pour qui la reçoit ? Comment peut se situer un·e médiateur rice dans cette activité de création, passée ou présente ? Cet article pose la médiation comme une activité partie prenante des processus créateurs en jeu.

Mots clés : création musicale ; médiation de la musique ; processus créateurs ; production artistique ; réception esthétique.
\end{abstract}

\begin{abstract}
How do you understand the making of music when you try to mediate it? How does the identification of the different actors who participate in the elaboration of a work give a hold on what a "work" is and what it represents for whom receives it? How can a mediator place him-herself in this creative activity, past or present? This article establishes mediation as an activity that is part of the creative processes at work.
\end{abstract}

Keywords: aesthetic reception; artistic production; creative processes; music mediation; musical creation. 
L'École d'hiver internationale en médiation de la musique de 2019 invitait à se pencher sur les liens entre création musicale et médiation de la musique. Les recherches que je mène depuis quelque temps déjà sur les processus créateurs en musique (Ravet 2015) ont rapidement fait émerger l'hypothèse suivante : travailler sur des processus et sur ce qu'est la créativité peut aider à penser ces liens et à agir comme médiateur-rice. S'en est suivi un ensemble d'interrogations liées entre elles : comment appréhender la fabrication de la musique lorsque l'on cherche à la médier ? En quoi l'identification des différents acteur'rice's qui participent à l'élaboration d'une œuvre donne-t-elle une prise sur ce qu'est une " œuvre » et ce qu'elle représente pour qui la reçoit ? Comment peut se situer un·e médiateur-rice dans cette activité de création, passée ou présente? La médiation ne fait-elle pas elle-même partie du processus créateur en jeu? En d'autres termes, ce texte se propose de montrer comment l'analyse de processus créateurs en musique, et en particulier l'analyse de leur dimension collective, éclaire la construction d'actions de médiation de la musique, notamment en contexte de «création".

Le matériau d'analyse à l'appui de cette réflexion provient d'observations in situ du " faire musical ». Les activités de médiation menées notamment par les étudiant·e·s du Master professionnel "Médiation de la musique " de l'Université Sorbonne Nouvelle et de Sorbonne Université depuis sa mise en place en 2015 éclairent ma pensée. Plus précisément encore, je mobilise deux types de terrain d'enquête explorés lors de précédentes recherches : l'une sur l'observation du travail orchestral et la manière dont un'e chef fe et des musicien 'ne $s$ élaborent une interprétation musicale (Ravet 2015) ; l'autre sur la manière dont le clarinettiste David Krakauer réinvente de la musique "traditionnelle » ou plutôt crée sa propre musique à partir du répertoire de la musique klezmer, en la croisant avec d'autres genres musicaux tels que le jazz ou le funk (Ravet 2011).

Analyser la musique en-train-de-se-faire, c'est-à-dire la manière dont se fabrique de la musique en tentant de saisir la participation de chacun'e des acteur-rice's et ce, au sein de multiples contextes musicaux, conduit à interroger ce qu'est la création en musique : je fais l'hypothèse qu'il s'agit là d'une piste fructueuse pour la médiation. Étudier ces processus donne un accès concret, appréhendable par différents types de public, à la manière dont sont conçues les musiques et ce qui s'apparente à de la création musicale. La présente réflexion souhaite ainsi montrer comment l'étude des processus créateurs en musique éclaire la pratique de médiateur rice de la musique et offre des outils spécifiquement en contexte de " création ». Elle se déclinera en deux temps : le premier temps consistera à préciser les contours de la création et des processus créateurs, tout en montrant qu'appréhender l'œuvre comme un faire collectif et comme un processus représente une approche riche et tangible pour agir en médiation ; le second temps précisera comment ce type d'approche offre une "prise " au’à la médiateur-rice pour développer des actions et quelles méthodes peuvent être mises en place ; cela portera à préciser en quoi la médiation s'apparente finalement aussi à un processus de création. L'article se donne ainsi pour objectif de montrer combien une conception sociologique des processus créateurs en musique et une réflexion sur ce qu'est la créativité permet de mettre en lumière des " prises " pour les pratiques de médiation de la musique. 


\section{L'EUURE COMME FAIRE COLLECTIF ET COMME PROCESSUS : UNE APPROCHE MÉDIATRICE}

Très concrètement, pour qu'un moment de musique vivante ait lieu et soit partagé, pour qu'une œuvre soit donnée à entendre, un vaste ensemble de personnes et d'activités est nécessaire : il faut des musicien·ne·s et a minima une " œuvre ". Si le concert mobilise des interprètes, ces dernier ère's ont besoin d'un instrument de musique (la voix, des instruments de lutherie, des instruments électroniques, etc.), dont elles·ils auront précédemment appris à jouer, ainsi qu'une œuvre à jouer, qu'elle se présente sous la forme d'une partition ou qu'elle soit composée par les participant·e·s en présence. Un cadre spatio-temporel défini s'avère aussi nécessaire pour que la performance ait lieu et, avant même le plus souvent, un temps pour travailler et répéter : un espace, ou une scène au sens le plus ouvert du terme, un temps donné consacré au concert, une information destinée à faire savoir qu'une performance aura lieu, notamment, tout cela demande la conjonction de multiples tâches coordonnées. Et le plus souvent, le tout vise la participation d'un public, convié d'une manière ou d'une autre à partager ce moment de musique vivante.

Rappeler ces quelques éléments éclaire la définition canonique en sociologie des arts des " mondes de l'art » proposée par Howard S. Becker ${ }^{1}$. Celle-ci pose que l'art est une action collective, qui repose sur le travail d'une multitude d'acteur.rice.s. Pour saisir ce qui s'élabore en art, il faut analyser tous les mécanismes qui concourent à la production des œuvres et de leur valeur. Il existe différents mondes de l'art et chaque monde de l'art se définit par l'ensemble des interactions nécessaire à la production sociale de la valeur artistique des œuvres reconnues comme œuvres d'art, par ce monde de l'art et parfois d'autres mondes. On voit ici l'importance de la désignation comme " œuvre " par un groupe d'acteur·rice.s.

Pour synthétiser dans ses grandes lignes la pensée de Becker, l'œuvre est le résultat d'un travail collectif de multiples participant·e.s au regard de son élaboration matérielle, mais aussi de sa confection symbolique, laquelle permet sa reconnaissance comme œuvre. Différents types de participant·e·s sont mobilisé·e·s dans les divers " mondes de l'art»: des " artistes » - reconnu·e's par ce monde de l'art et qui apportent une contribution indispensable à l'existence de l'œuvre et à sa reconnaissance comme telle - jusqu'aux " personnels de renfort "-constitués de personnes substituables entre elles, mais dont l'action est indispensable à l'avènement de l'œuvre. Cela comprend aussi un grand nombre d'intermédiaires dont il est souvent difficile de situer la contribution sur le continuum de celles des « artistes » et des " personnels de renfort " (ainsi de la critique, du·de la producteur.rice d'un film, par exemple) $)^{2}$. Il demeure tout aussi difficile de savoir où arrêter la participation de tel ou telle à la fabrication de l'œuvre (ainsi qu'en témoigne, par exemple, le générique de fin d'un

$1 \quad$ Une autre définition canonique d'un monde de l'art s'offre comme le « réseau de tous ceux dont les activités, coordonnées grâce à une connaissance commune des moyens conventionnels de travail, concourent à la production des œuvres qui font précisément la notoriété du monde de l'art » (Becker [1982]1988, p. 22).

2 Cf. la série de travaux récents sur les intermédiaires et leur rôle effectif dans la création de l'œuvre, dont Lizé, Naudier et Roueff 2011. 
film). Les « mondes de l'art» se caractérisent par des processus créateurs, caractérisés par l'action de réseaux au sein desquels les personnes se coordonnent grâce à des « conventions » communes à partir d'une « idée » ou d'un « projet » initial.

Dans cette appréhension de la fabrication des œuvres, le·la médiateur·rice apparaît donc comme un.e acteur rice, parmi d'autres, qui contribue à cette confection. Par conséquent, plutôt que d'aborder l'œuvre comme un " déjà là » et poser implicitement son existence pleine et entière avant sa médiation et sa réception, la considérer comme œuvre en construction, en train-de-se-faire, donne une place de choix pour la médiation.

Pour cela, un changement de regard est nécessaire par rapport aux usages habituels. Il convient de considérer les œuvres comme des pratiques objectivées ou des objets pratiqués. Elles apparaissent toujours comme telles, en dernière instance, à qui chausse les lunettes de sociologue que le·la médiateur rice est ici invité e à emprunter. Certes, les œuvres subsistent sous forme de traces matérielles, ont une organisation, une consistance, présentent des résistances aux multiples appréhensions qui peuvent en être faites ; mais ces traces matérielles, ces organisations, ces consistances n'existent symboliquement que dans leur relation avec les acteur-rice-s qui les manipulent ou les commentent. Pour restituer le ou les sens de l'œuvre, le·la médiateur-rice gagne(rait) dès lors à s'efforcer de référer les structures « internes " de cette dernière à un "faire " concret et contextualisé (comme y invitent, de manière générale, sans évoquer les actions de médiation : Laborde 2012 ; Kirchberg et Robert 2014).

On aura remarqué que la réflexion mobilise tour à tour les expressions de "processus de création ", "processus créateurs » et " créativité ». Il est vain de tenter de les décliner et de les définir successivement dans l'espace textuel imparti. Les enjeux de définition et les questionnements qu'ils suscitent sont multiples ${ }^{3}$. Il est toutefois important de pointer plusieurs aspects essentiels.

En premier lieu, il est nécessaire d'interroger un tant soit peu le terme de "création ». Un bref retour sur l'histoire du terme rappelle qu'il s'est laïcisé à partir du XIve siècle avec le sens d' " action d'établir une chose pour la première fois " (Rey [1992]1994, p. 525-526). Au XVIII siècle, le créateur est l'« auteur d'une chose nouvelle ». Dans les arts, par la suite, le terme renvoie à " action de créer ", lequel vient de la signification d'origine religieuse, tirer du néant ou faire surgir du néant, par opposition à l'« imitation».

En deuxième lieu, le matériau d'enquête mobilisé ici mérite une précision. Qu'il s'agisse du travail entre un'e chef'fe et des instrumentistes ou celui d'un musicien comme David Krakauer, dans ces deux cas, des figures d'autorité marquent le travail collectif entre interprètes : soit en tant qu' " artiste » reconnu comme tel dans certains milieux du jazz et des musiques improvisées, à propos de David Krakauer, soit en tant que chef-fe d'orchestre, pour les ensembles observés ; c'est-à-dire, dans les deux cas, comme "créateur ", c'est-à-dire comme personne dotée d'une autorité créatrice.

3 Voir, par exemple, la série de conférences internationales Tracking the Creative Process in Music impulsée par Nicolas Donin. 
Dans autorité, étymologiquement, il y a " auteur » ${ }^{4}$. Mais ces deux types de figures créatrices se distinguent ou se posent en décalage par rapport au type d'artiste auquel l'on songe spontanément en réfléchissant à la "création " dans le domaine de la musique dite savante. La figure du « compositeur » est généralement à laquelle on se réfère. Or, c'est précisément ce décalage qui intéresse, car il permet d'en apprendre beaucoup sur la " création » de musiques propres à différents genres musicaux.

En troisième lieu, le terme "création " lui-même comporte une dimension qui peut apparaître comme figée : on envisage, on parle d'" une " création, considérée comme un produit, une forme (en partie au moins) aboutie. Parler de " processus créateurs " conduit plutôt à mettre l'accent sur ce qui produit l'œuvre au terme d'un processus (que d'aucun·e nommerait " processus de création »). Plus radicalement, on peut concevoir l'œuvre comme un processus toujours ouvert, en devenir, en écho notamment à Umberto Eco et son « œuvre ouverte » (Eco [1965]2015). Cette position conduit à étudier des " processus créateurs ", où le " produit " peut être considéré comme la cristallisation provisoire d'un processus toujours en cours.

La construction d'une interprétation orchestrale l'illustre. L'exemple peut paraître contre-intuitif, là encore : l'œuvre portée par un· e compositeur·rice est déjà «créée », au sens où une première occurrence publique a eu lieu qui a donné à entendre l'œuvre une première fois. On peut dès lors considérer que les interprétations suivantes participent d'une perpétuelle " recréation ", quand d'autres conceptions évacueront l'idée même de création à ce stade. Cependant, en décalant le regard, une autre perception de la création surgit au sein du travail orchestral. L'ethnographie permet de comprendre la manière dont se constituent collectivement des interprétations musicales et quelle part chacun·e des participant·e·s prend au processus créateur.

En particulier, la conception assez usuelle selon laquelle le « chef » décide de tout à partir d'un projet défini par avance se trouve ainsi mise en question ${ }^{5}$. À l'observer de plus près, la créativité s'avère partagée entre chef·fe et musicien·ne·s. Elle se loge dans et surgit de la pratique routinière de la répétition. J'ai ainsi pu montrer comment : 1) le·la chef·fe prend appui sur des relais (notamment le " premier violon » et les chef·fe's de pupitre) ; 2) la manière dont des formes d'autorégulation entre musicien·ne.s caractérisent le travail et le son d'un pupitre ; 3) le rôle des interventions spontanées et la réaction des chef·fe·s aux propositions des musicien·ne·s, qu'elles soient verbales ou instrumentales ; 4) l'existence de formes de coopération, lesquelles se construisent au cœur de la temporalité des répétitions successives et du travail mené au sein d'un ensemble sur plusieurs années, ce qui n'exclut pas des résistances et des enjeux de pouvoir majeurs. Deux résultats majeurs marquent la recherche. D'une part, l'autorité au sein d'un orchestre se révèle ainsi sans cesse négociée et renégociée au jour le jour entre l'ensemble des participant·e·s. D'autre part, la créativité - loin de n'être le fruit de l'action que d'une seule personne, celle que l'on

\footnotetext{
4 Au sens étymologique d'auctoritas, le fait d'être auteur, qui fonde le pouvoir accordé au chef.

5 Sur les différentes représentations de la figure du chef d'orchestre, de l'image du dictateur à l'histrion inutile, et leur mobilisation au long de l'histoire, voir Buch 2002.
} 
appelle l'artiste ou qui endosse la figure du " créateur » - cette créativité surgit en partage entre tou·te.s les participant·e.s à l'action, lesquel·le·s y contribuent chacun·e à sa manière (Ravet 2015).

Un autre exemple vient à l'appui de l'analyse de l'œuvre en termes de processus créateurs. David Krakauer, clarinettiste new-yorkais, formé à la musique dite classique et au jazz, participe d'une réinvention de la musique klezmer, dont il explique que la transmission a été interrompue en raison des événements politiques du $\mathrm{xx}^{\mathrm{e}}$ siècle. Comment crée-t-il sa propre musique inspirée de cette tradition ? Il s'appuie sur des modes de jeu clarinettistiques construits par l'écoute phonographique, tels que la manière d'émettre et d'articuler le son à la clarinette, l'usage de broderies, les Krechts (sanglots dans la voix) etc., outre des « airs traditionnels » qu'il reprend. Il les combine avec des modes de jeu personnels, comme l'usage des suraigus, influencé par le jazz qu'il a pratiqué un temps aux côtés de John Zorn, des musiques improvisées de manière générale, mais aussi - par la suite - en dialoguant avec le sampling de DJ Socalled ou en croisant avec la musique funk de Fred Westley. Il résulte de ces multiples influences une œuvre en devenir que l'on peut appréhender et à laquelle on peut donner accès précisément en examinant ses sources et leurs combinaisons. Tirer les fils de ces diverses influences permet de mieux saisir comment elle sonne et y déceler, si ce n'est un sens, tout au moins des pistes, pour appréhender cette musique et se familiariser avec elle.

Un courant de travaux se penche aujourd'hui en musicologie sur ces processus de création, en se situant par rapport aux recherches en génétique de la musique. "Créer à plusieurs mains ", pour reprendre le titre du numéro de Genesis dirigé par Nicolas Donin et Daniel Ferrer (2015) montre le rôle des ami·e·s, relecteurs $\cdot$ rices, éditeurs rices, etc. dans la composition des manuscrits chez les écrivain·e·s et d'autres artistes. La " création " musicale fait intervenir des acteur-rice's variables selon les univers et les genres musicaux : des compositeur·rice's et des interprètes, des ingénieur·e·s du son, des réalisateurs musicaux à l'Ircam, etc. ; des artistes-créateur·ice·s parfois crédité·e·s comme compositeur·rice·s - comme David Krakauer pour lequel il ne s'agit pas de la seule attribution, ni parfois de l'attribution principale - mais aussi des collectifs, des groupes, etc.

C'est tout l'intérêt d'une approche en termes de processus créateurs en musique, dont l'orientation s'avère plus large que celle de processus de création musicale à strictement parler, car elle propose une ouverture à la prise en compte de la spécificité des différents genres musicaux (hors musique dite savante, notamment). Elle souligne la multiplicité des participations possibles à la création des différents types d'œuvres évoqués (avec ou sans " compositeur·rice " désigné·e comme tel·le, en particulier). Pour qui agit en médiation, il y a bien là une approche possible de la création : la dimension concrète, effective de l'« œuvre ", d'un côté, la multiplicité des participant·e.s et des types de participation, de l'autre - ces deux dimensions donnent des « prises » pour pouvoir appréhender l'œuvre et la création. Je vais m'y arrêter maintenant plus précisément en examinant comment. 
LES PROCESSUS CRÉATEURS COMME « PRISE » : ENJEUX ET MÉTHODES EN MÉDIATION

Comment saisir ces « prises " possibles pour la médiation en contexte de création? Et pourquoi mobiliser le concept de " prise " ? Une précision théorique s'avère de nouveau nécessaire. Ce concept de " prise » est entendu au sens à la fois d'accroche, de saisie de ce qui fait saillance dans l'œuvre, d'attachement possible à quelque chose. On peut ici se référer à la pensée d'Antoine Hennion $(1993,2004)$ et des recherches qu'il a menées avec Sophie Maisonneuve et Émilie Gomart sur la musique dite classique autour des " figures de l'amateur » (2000). "La musique est médiation », affirme Hennion : elle est portée par des humains et des non-humains ; elle est toute entière pratiques, objets, dispositifs (mixtes) ; elle est par la scène, l'instrument, la partition, le disque, écrit-il alors (Hennion 1993). Par la suite, le chercheur a largement développé le concept de «prise » en montrant comment les « amateurs » de musique, au sens de ceux qui aiment, goûtent et apprécient, construisent des " attachements » au travers en particulier de la matérialité du disque, ce qui le conduit avec ses coauteures à parler de « discomorphose» :

La notion de prise permet de rendre compte de l'émotion et des « coups de cœur » tout en tenant compte des degrés de familiarisation et de connaissance. Loin que cette notion nous fasse verser dans le relativisme ou l'individualisme, elle nous permettra d'aborder la construction d'une relation spécifique à la musique classique, celle à laquelle ouvrent ses supports et formats modernes : elle nous permet, par l'attention à la relation (inter)active sujet-objet, de rendre compte du mécanisme de la médiation et d'étudier ces formats modernes comme médiateurs décisifs de la musique classique. (Hennion, Maisonneuve et Gomart 2000, p. 126)

La musique apparaît plus généralement comme «technique collective de fabrication de soi » (ibid., p. 43).

Dès lors, comment saisir la dimension effective de l'œuvre, le ou les processus créateur.s qui la caractérise $n t$, pour faire médiation? De manière pragmatique, une telle action médiatrice consiste à identifier et à présenter l'apport des divers participant $\cdot e \cdot s$ au faire collectif, à préciser leurs contributions matérielles - leur apport très concret au faire, comme de produire tel son ou d'assurer, par exemple, le soutien harmonique - et leurs contributions symboliques - c'est-à-dire ce qui va permet d'accorder un statut d'œuvre dans tel ou tel contexte au faire collectif, comme ce qui va la rattacher à tel ou tel univers musical. Mettre ces participations en lien avec rôle social de l'acteur rice au sein du processus, ainsi qu'avec la manière de sonner de l'œuvre, dans un univers de référence qui dispose de ses propres conventions, ses codes et ses contraintes, voilà qui peut permettre de proposer à autrui de quoi s'approcher de l'œuvre et peut-être de se l'approprier, de la faire sienne dans l'acte de réception. Le jeu au trombone de Fred Westley, par exemple, signifie dans l'univers sonore du funk et rattache telle pièce de tel album « de » David Krakauer à cet univers musical. En sens, il véhicule de la signification.

Mettre en place ce type de médiation porte donc à articuler l'analyse des contributions concrètes et symboliques des acteur-rice-s en présence avec celle du matériau musical et à appréhender l'œuvre comme espace de signification, notamment 
pour celles et ceux qui l'élaborent : cela "veut dire ", ou plutôt, cela fait sens pour qui, pourquoi, comment, notamment pour les personnes qui fabriquent l'œuvre. L'objectif demeure commun à de nombreuses approches en médiation des arts et de la musique : il s'agit de donner des clés à celles et ceux qui la reçoivent, non pas tant pour affirmer « cela veut dire ceci », qu'en observant la manière dont l'œuvre se construit, matériellement et esthétiquement, pour celles et ceux qui la fabriquent... et donc qui la reçoivent également. Il s'agit là d'autant de « prises » pour donner accès à une « création ».

Pour revenir à l'approche de la "création » déclinée lors du premier temps de la réflexion, je propose de tirer un fil qui se distingue sensiblement de la conception de la création comme processus ex nihilo. Les travaux de Donald W. Winnicott sur le développement psychologique des jeunes enfants (voir Leroy 2013), notamment, montrent que la créativité contribue à construire l'individu ; elle surgit au cœur de la monotonie : au gré des microvariations, un espace potentiel de jeu s'ouvre où tend à se constituer, dans le social, l'identité psychique de l'individu.

Un parallèle s'offre à faire en musique : la tension entre créativité et routine - cette manière dont quotidien et exception s'entremêlent - irrigue les processus créateurs, comme 1'explique Anselm Strauss :

C'est par un processus cyclique que la routine se transforme en créativité et en innovation et qu'à leur tour, celles-ci redeviennent de la routine. Au sens propre du terme, la routine n'est pas simple « routine » et la créativité ou l'innovation ne sont en aucun cas coupées de leur racine, la routine. (Strauss 1994, p. 365 ; souligné par l'auteur)

Cela prend tout son sens en musique, notamment dans la " répétition " qui permet qu'advienne une création lors d'un concert, pour reprendre notre exemple initial. Et cela irrigue notre proposition du côté de la médiation : saisir des microvariations, des déplacements par rapport à une configuration esthétique ; dit autrement, mettre en lumière le socle de routines qui sous-tend la créativité, c'est-à-dire les codes usuels, les conventions existantes, voire les canons en place, et les pas de côté, les propositions inédites ou décalées ; cela revient à dévoiler le côté artisanal de la création. C'est à mon sens une autre piste d'action pour qui cherche à médier une œuvre.

Quelle place, finalement, peut occuper un·e médiateur-rice dans un tel processus? Quel type de "dispositif " inventer ou proposer ? Le terme de dispositif engage lui-même une réflexion, discutant notamment la pensée foucaldienne (Foucault 1975), qui dépasse le cadre de cet article. À tout le moins, précisons que l'on peut entendre par « dispositif de médiation »

la conception du cadre dans lequel les interactions de médiation se déroulent (y compris leur propre rôle à l'intérieur de cet espace, ce qui explique qu'on les identifie eux-mêmes comme acteurs de la médiation). Concevoir un tel cadre peut nécessiter des compétences différentes selon les cas, mais impose que soient spécifiés, de manière plus ou moins explicite : les publics visés, le cadre spatio-temporel dans lequel la médiation se déroule, le type de contenu qui doit être transmis, utilisé ou produit, etc. Cette conception peut être le produit d'une démarche collective effectuée en interne ou bien d'une démarche en partenariat (collectivités locales, structures associatives, autres acteurs publics...). (Aubouin, Kletz et Lenay 2010, p. 3) 
Les recherches de Laurent Fleury sur un dispositif de « correspondants » mis en place au Centre Beaubourg dans les années 1970-1980 (comme amis-passeurs auprès des publics) permettent de préciser l'importance du concept de dispositif (Fleury 2008). L'auteur se réfère aux travaux de Lucien Karpik (1996) distinguant dispositif de confiance et dispositif de jugement :

Les dispositifs de médiation opèrent donc un pouvoir de structuration des pratiques en transformant des façons de vivre des relations à l'art, des expériences culturelles, voire des pratiques artistiques. Ces effets peuvent être thématisés en un double impact. Le premier, de type normatif, se trouve à l'origine de transformations dans la fréquentation. Un second, plus cognitif, influence les modes de représentations de la relation de l'individu à l'art et structure les registres d'identification à l'institution. (Fleury 2008, p. 21)

Concrètement, pour en revenir aux propositions à propos de la fabrication de l'œuvre comme fil pour construire une médiation, que peut faire le·la médiateur·rice? Tout d'abord, il peut s'agir de construire un dispositif pour permettre de rencontrer celles et ceux qui font l'œuvre : observer les étapes d'élaboration, rencontrer les différent·e·s participant·e·s à l'œuvre, les écouter relater leur action et raconter la manière dont elles et ils ont pris part à l'élaboration de l'œuvre. Dans un deuxième temps, de manière complémentaire, mais il peut tout aussi bien s'agir d'une proposition en soi, on peut imaginer donner accès aux «traces » de la participation à l'œuvre ; partitions, brouillons, maquettes successives d'un projet musical... L'étape suivante réside dans l'analyse de la forme sonore de cette trace au regard de l'univers de signification construit par les acteur. rice's en contexte.

Ce travail est multiforme et multimodal. Il nécessite la prise en compte des publics dans les choix des approches en médiation. Se préoccuper de qui vient précisément faire public, des expériences, du rapport à la musique, voire des besoins des auditeur-rice's qui composent ce public ici et maintenant (si tant est que l'on puisse en partie connaître ces besoins ou aspirations), voilà une tâche d'importance pour choisir comment aborder tel ou tel processus créateur, comment le déplier pour permettre $\mathrm{au} \cdot a ̀$ la spectateur rice de le « saisir » d'une manière ou d'une autre. Trois dimensions d'ordre épistémologique ressortent de ce travail multiforme et multimodal :

1. La première relève d'un travail de traduction ou de déconstruction de la construction : déplier la multiplicité des contributions, exposer le cheminement des rencontres et des collaborations pour mieux appréhender ce qui fait "œuvre " ici et maintenant pour les musicien·ne·s ou participant·e $s$ en présence, ainsi que la signification partagée donnée à cette élaboration ;

2. Une deuxième dimension se déploie dans le travail d'élaboration d'un espace de rencontre du public considéré avec les acteur·rice·s présent·e·s ou passé·e·s du processus créateur examiné, ou à distance (tel·le·s les compositeur·rice·s des siècles passés);

3. Enfin, une troisième dimension émerge du travail de mise en exergue de prises possibles pour les publics dans le matériau sonore et/ou d'accompagnement du conflit esthétique : qu'est-ce qui vient heurter les représentations existantes des publics? Comment s'approprier une proposition artistique, faire avec, la modeler à 
son tour? Et ce, à la fois en tant que public et en tant que médiateur·rice, dans une activité à destination de soi et d'autrui ?

Pourquoi ici parler de conflit esthétique ? Quel rapport avec une médiation en contexte artistique et/ou culturel ? Quelle part y prend une personne en situation de médiation? Bruno Péquignot (2007) explique qu'une médiation a toujours lieu dans une institution, qui définit des croyances et des modes de conduite. La médiation n'est pas communication, entendue comme action de transmettre d'un'e émetteur-rice à un·e récepteur-rice une information, tout en faisant en sorte qu'il y ait le moins de perte ou de bruit possible dans cette transmission. L'action du-de la médiateur.rice en contexte artistique et/ou culturel consiste à faire accéder le public à la dimension spécifiquement esthétique d'une œuvre, ce qui est aussi faire accéder à une démarche de création au sein d'une institution. L'artiste propose une manière de voir, au sens large. Il revient à la personne qui réalise une médiation de " faire voir ce qu'on ne voit pas dans ce que l'on voit " et d'amener le public à interroger cet autrement. Il s'agit d'accompagner vers la découverte de cet autrement, qui peut entrer en conflit avec nos propres représentations. Cela ne consiste pas à tenter de statuer impérativement sur ce que recouvre cet autrement ou à chercher à lui donner une signification univoque, mais bien au contraire à ouvrir les possibles pour qu'autrui s'en saisisse et fasse son propre chemin dans cette rencontre. Pour le·la médiateur-rice, il s'agit donc d'autant moins d'affirmer " cela veut dire ceci » face à une ouvre, comme évoqué précédemment, que de viser la figure du "spectateur émancipé », telle que proposée par Jacques Rancière (2008). Familiariser un public avec les logiques des processus créateurs et l'accompagner dans le conflit éventuel causé avec ses propres représentations ouvre un espace d'interprétation des œuvres. Que ce public soit ou non familier de l'univers esthétique considéré, qu'il y soit peu ou pas initié, cet espace d'interprétation peut émerger à partir des expériences vécues et singulières de chaque spectateur-rice, mais aussi, d'autant plus que les repères sont bousculés, au-delà de celles-ci.

Afin d'éclairerces propositions, jeciterai en dernier exemple celuide David Krakauer, observé lors d'un atelier dans le cadre du Festival Villes des musiques du monde en 2006 : le musicien a entrepris de jouer un air traditionnel, de le contextualiser en proposant des pistes d'interprétation, tout en répondant à des questions sur la culture yiddish ; puis il a montré des modes de jeu, avant de faire participer rythmiquement le public présent à l'aide de percussions corporelles; il s'est ensuite mis à improviser

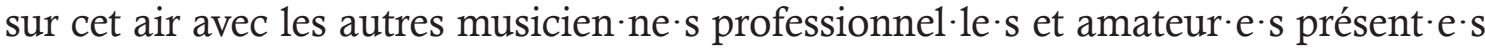
jusqu'à ce qu'une sorte de participation dansée collective incluant l'ensemble du public émerge et se développe. La médiation était réalisée par l'artiste lui-même en coopération avec la personne qui accompagnait le public de cet atelier-découverte auquel participaient de nombreux enfants et qui se déroulait en plein air devant un centre social. Ici, la médiation s'est traduite via le mouvement, dans le son et par la rencontre forgeant un collectif éphémère. Cela a été rendu possible par l'intense énergie communicative de l'artiste qui partageait un répertoire peu connu du public, lequel pouvait entrer en résonance avec les répertoires et les références culturelles très différenciées des personnes en présence, dans l'accompagnement bienveillant de la personne chargée du public visé. L'œuvre prenait ainsi sens et existence, ici et 
maintenant, la médiation - entendue comme rencontre sociale et esthétique - faisant pleinement partie du processus créateur.

Un dernier retour théorique peut éclairer et ouvrir encore des pistes complémentaires pour la médiation des phénomènes esthétiques. Becker pointe le « Principe de l'Indéterminabilité Fondamentale de l'Euvre d'Art », selon lequel : "Ce que l'œuvre est, sans être le moins du monde arbitraire, est sujet à de grandes variations et ne peut jamais être établi de façon définitive, qui soit dictée par sa nature physique [...]. "L'œuvre elle-même" n'est isolée qu'en vertu d'un acte collectif de définition " (Becker 2003, p. 30). On ne peut ni dire le " tout » de l'œuvre ni l'appréhender dans sa totalité. Toute œuvre a une trajectoire, un itinéraire, un parcours propre - certains parlent même de " biographie " (Lahire 2015) ou de " carrière " (Menger 2010) à propos précisément des œuvres et pas seulement des artistes. Celle-ci se transforme au gré de ses multiples réceptions, appropriations et recréations.

Ceci revient à dire que chaque œuvre consiste finalement en une succession d' « occurrences » ou de « figures » (Dupont 2011, p. 147-148) plus ou moins précises et plus ou moins différentes les unes des autres, que l'interprétation au moment $t$ donné, voire les interprétations successives au grès de la fréquentation des œuvres dans le temps de chaque auditeur-rice, spectateur-rice, contribue à construire, fabriquer, élaborer. Cette conception éloigne d'une vision purement essentialiste et immuable de l'œuvre. Elle se pose contre, comme le dit Pierre Bourdieu, l'illusion de la « constance du nominal » (Bourdieu 1986, p. 71) : les œuvres étant presque toujours affublées de noms propres, elles seraient supposées " naître " chef-d'œuvre par elles-mêmes et le demeurer dans le temps. L'étude des processus créateurs met à distance l'idée de "naissance " comme œuvre, par essence, pour montrer l'effectivité collective du devenir œuvre : c'est parce que diverses personnes travaillent à la faire œuvre, qu'elle devient une ouvre, à la fois matériellement (dans les interactions pratiques) et symboliquement (dans la reconnaissance sociale en tant qu'œuvre). Quant à la pérennité des œuvres dans le temps, c'est là une autre piste possible pour la médiation : réfléchir à la manière dont on écrit l'histoire de la musique ou des arts, comment s'élabore ce récit collectif porté par des voix singulières... et quelle place y prennent les médiateur'rice's.

En ce sens, toute médiation - en construisant des ponts, en tissant des liens entre artiste et public - participe à l'effectivité de l'œuvre. À ce titre, la créativité de la médiation se situe notamment dans l'ouverture d'un espace de réception pour l'œuvre en train de naître, dans le jeu ouvert entre personnes au sein de l'espace interprétatif ainsi créé. Pour paraphraser de nouveau Hennion selon lequel la musique est toute entière médiation, la création est médiation ; le terme "médiation " est ici entendu à la fois au sens mobilisé par Hennion, mais aussi pour désigner un type de pratique professionnelle et de champ disciplinaire d'action ; la création est faite de médiations et l'acte de créer est déjà un acte de médiation. En retour, la médiation - cette fois entendue en tant que pratique professionnelle et champ disciplinaire d'action participe de la création. 
Pour aborder la création en médiation, je suis partie de l'hypothèse selon laquelle travailler sur des processus et sur ce qu'est la créativité peut aider à penser et à agir comme médiateur-rice. Cela éclaire un triple entrecroisement entre création et médiation, en musique tout comme dans d'autres domaines artistiques : d'une part, la médiation se révèle intrinsèque à l'acte de créer, à la performance ou à la représentation ; d'autre part, les processus créateurs représentent une " prise " pour la mise en place d'une action de médiation ; enfin, la médiation participe aussi d'un processus de création.

Cheminant au travers d'un certain nombre de précisions théoriques sur la création, les processus créateurs, l'œuvre comme faire collectif, le concept de " prise ", notamment, et à l'aune d'exemples issus d'enquêtes de l'observation sur terrain de la musique en-train-de-se-faire, j'ai mis en exergue quelques « prises » possibles - pour qui fait œuvre de médiation - à partir de ma proposition initiale : identifier et faire rencontrer les participant·e·s à la création ; distinguer leur contribution à l'œuvre, matérielle et symbolique, en lien avec la manière dont sonne l'œuvre ; offrir ainsi des clés d'écoute tangibles, incarnées dans des personnes et des objets.

À la fois théoriquement méthodologiquement, la prise en compte des processus créateurs en musique permet donc d'élaborer des stratégies de médiation de la musique adaptées à différents types de publics et de cibler ce à quoi on souhaite donner accès. Ainsi, l'expérience des processus de création est particulièrement riche pour les publics non-initiés, si tant est que l'on vise une appropriation ouverte à de multiples interprétations, voire une "émancipation » de ces spectateur·rice·s. La médiation de la musique, telle qu'envisagée ici, se définit pleinement comme un processus créateur collaboratif entre médiateur·rice·s, interprètes et compositeur·rice·s, publics et œuvre. Elle est créatrice d'une " signification " musicale, au sens le plus large donné à ce terme. C'est là où elle fait œuvre de créativité : en proposant un espace interprétatif ouvert aux multiples expériences des publics.

\section{BIBLIOGRAPHIE}

Aubouin, Nicolas, Frédéric Kletz, et Olivier Lenay (2010), « Médiation culturelle. L'enjeu de la gestion des ressources humaines ", Culture études, vol. 1, no 1, p. 1-12, https://www.cairn.info/revueculture-etudes-2010-1-page-1.htm, consulté le 8 septembre 2020.

Becker, Howard S. ([1982]1988), Les mondes de l'art, Paris, Flammarion.

Becker, Howard S. (2003), Paroles et musique, Paris, L'Harmattan.

Bourdieu, Pierre (1986), "L'illusion biographique ", Actes de la recherche en sciences sociales, vol. 6263, p. 69-72, https://www.persee.fr/doc/arss 0335-5322 1986 num 6212317 , consulté le 17 septembre 2020.

Buch, Esteban (2002), «Le chef d'orchestre. Pratiques de l'autorité et métaphores politiques ", Annales. Histoire, Sciences Sociales, $57^{\mathrm{e}}$ année, $\mathrm{n}^{\circ}$ 4, p. 1001-1028, https://www.cairn.info/revue-annales2002-4-page-1001.htm, consulté le 8 septembre 2020.

Donin, Nicolas, et Daniel Ferrer (dir.) (2015), “Créer à plusieurs mains » (dossier), Genesis, n 41, https://journals.openedition.org/genesis/1193, consulté le 8 septembre 2020.

Dupont, Maïlys (2011), Le bel aujourd'hui. Bach ou Boulez, des œuvres à faire, Paris, Cerf.

Eco, Umberto ([1965]2015), L'auvre ouverte, Paris, Le Seuil. 
Fleury, Laurent (2008), «L'influence des dispositifs de médiation dans la structuration des pratiques culturelles. Le cas des correspondants du Centre Pompidou ", Lien social et Politiques, n 60 (automne), p. 13-24, https://www.erudit.org/fr/revues/1sp/2008-n60-1sp2511/019442ar/, consulté le 8 septembre 2020.

Foucault, Michel (1975), Surveiller et punir. Naissance de la prison, Paris, Gallimard.

Hennion, Antoine (1993), La passion musicale. Une sociologie de la médiation, Paris, Métailié.

Hennion, Antoine (2004), "Une sociologie des attachements. D'une sociologie de la culture à une pragmatique de l'amateur ", Sociétés, vol. 3, no 85, p. 9-24, https://www.cairn.info/revue-societes2004-3-page-9.htm, consulté le 8 septembre 2020.

Hennion, Antoine, Sophie Maisonneuve, et Émilie Gomart (2000), Figures de l'amateur. Formes, objets, pratiques de l'amour de la musique aujourd'hui, Paris, La Documentation française.

Karpik, Lucien (1996), "Dispositifs de confiance et engagements crédibles », Sociologie du travail, $38^{\mathrm{e}}$ année, $\mathrm{n}^{\circ} 4$ (octobre-décembre), p. 527-550, https://www.persee.fr/doc/sotra 0038-0296 1996 num 3842284 , consulté le 8 septembre 2020.

Kirchberg, Irina, et Alexandre Robert (dir.) (2014), Faire l'art. Analyser les processus de création artistique, Paris, L'Harmattan.

Laborde, Denis (2012), «L'ethnomusicologie sert-elle encore à quelque chose?», dans Emmanuel Brandl, Cécile Prévost-Thomas Cécile et Hyacinthe Ravet (dir.), 25 ans de sociologie de la musique en France, «Tome 2. Ancrages théoriques et rayonnement international », Paris, L'Harmattan, p. 197-208.

Lahire, Bernard (2015), Ceci n'est pas un tableau. Essai sur l'art, la domination, la magie et le sacré, Paris, La Découverte.

Leroy, Jean-Luc (2013), " La musique créative au service de la construction sociale et identitaire. Point de vue à partir de la théorie de Winnicott », colloque "L'intégration et la socialisation à travers des médiations culturelles et artistiques », Université Paris Est Créteil, 11-12 février 2013.

Lizé, Wenceslas, Delphine Naudier, et Olivier Roueff (2011), Intermédiaires du travail artistique. À la frontière de l'art et du commerce, Paris, La Documentation française.

Menger, Pierre-Michel (2010), «Le travail à l'œuvre. Enquête sur l'autorité contingente du créateur dans l'art lyrique ", Annales. Histoire, Sciences sociales, 65e année, no 3 (mai-juin), p. 743-786, https://www.jstor.org/stable/40801644, consulté le 8 septembre 2020.

Péquignot, Bruno (2007), "Sociologie et médiation culturelle ", L'Observatoire, vol. 32, no 2, p. 3-7, https://www.cairn.info/revue-1-observatoire-2007-2-page-3.htm, consulté le 8 septembre 2020.

Rancière, Jacques (2008), Le spectateur émancipé, Paris, La fabrique.

Ravet, Hyacinthe (2011), " David Krakauer. "My Grandmother's Voice" and the Reinvention of Klezmer Music / David Krakauer. "Die Stimme meiner Großmutter" und die Neuerfindung der Klezmer-Musik ", JMB (Jewish Museum Berlin) Journal, no 4, p. 24-28.

Ravet, Hyacinthe (2015), L'orchestre au travail. Interactions, négociations, coopération, Paris, Vrin.

Rey, Alain (dir.) ([1992]1994), "Créer », Dictionnaire historique de la langue française, Paris, Le Robert, p. $525-526$.

Strauss, Anselm (1994), «L'influence réciproque de la routine et non-routine dans l'action », dans JeanClaude Passeron et Pierre-Michel Menger (dir.) L'art de la recherche. Essais en l'honneur de Raymonde Moulin, Paris, La Documentation Française, p. 349-366. 